\title{
Aging Rate of Spin Glasses from Simulations Matches Experiments
}

\author{
M. Baity-Jesi, ${ }^{1,2}$ E. Calore, ${ }^{3}$ A. Cruz, ${ }^{4,2}$ L. A. Fernandez, ${ }^{5,2}$ J. M. Gil-Narvion, ${ }^{2}$ A. Gordillo-Guerrero, ${ }^{6,7,2}$ \\ D. Iñiguez, ${ }^{2,8}$ A. Maiorano, ${ }^{9,2}$ E. Marinari, ${ }^{10}$ V. Martin-Mayor, ${ }^{5,2}$ J. Moreno-Gordo, ${ }^{2,4}$ A. Muñoz-Sudupe, ${ }^{5,2}$ \\ D. Navarro, ${ }^{11}$ G. Parisi, ${ }^{10}$ S. Perez-Gaviro, ${ }^{12,2,4}$ F. Ricci-Tersenghi, ${ }^{10}$ J. J. Ruiz-Lorenzo, ${ }^{13,7,2}$ S. F. Schifano, ${ }^{14}$ \\ B. Seoane, ${ }^{15,2}$ A. Tarancon, ${ }^{4,2}$ R. Tripiccione, ${ }^{3}$ and D. Yllanes ${ }^{16,2, *}$ \\ (Janus Collaboration) \\ ${ }^{1}$ Department of Chemistry, Columbia University, New York, New York 10027, USA \\ ${ }^{2}$ Instituto de Biocomputación y Física de Sistemas Complejos (BIFI), 50018 Zaragoza, Spain \\ ${ }^{3}$ Dipartimento di Fisica e Scienze della Terra, Università di Ferrara e INFN, Sezione di Ferrara, I-44122 Ferrara, Italy \\ ${ }^{4}$ Departamento de Física Teórica, Universidad de Zaragoza, 50009 Zaragoza, Spain \\ ${ }^{5}$ Departamento de Física Teórica, Universidad Complutense, 28040 Madrid, Spain \\ ${ }^{6}$ Departamento de Ingeniería Eléctrica, Electrónica y Automática, Universidad de Extremadura, 10003 Cáceres, Spain \\ ${ }^{7}$ Instituto de Computación Científica Avanzada (ICCAEx), Universidad de Extremadura, 06006 Badajoz, Spain \\ ${ }^{8}$ Fundación ARAID, Diputación General de Aragón, Zaragoza, Spain \\ ${ }^{9}$ Dipartimento di Fisica, Sapienza Università di Roma, I-00185 Rome, Italy \\ ${ }^{10}$ Dipartimento di Fisica, Sapienza Università di Roma, INFN, Sezione di Roma 1, and CNR-Nanotec, I-00185 Rome, Italy \\ ${ }^{11}$ Departamento de Ingeniería, Electrónica y Comunicaciones and I3A, Universidad de Zaragoza, 50018 Zaragoza, Spain \\ ${ }^{12}$ Centro Universitario de la Defensa, Carretera de Huesca s/n, 50090 Zaragoza, Spain \\ ${ }^{13}$ Departamento de Física, Universidad de Extremadura, 06006 Badajoz, Spain \\ ${ }^{14}$ Dipartimento di Matematica e Informatica, Università di Ferrara e INFN, Sezione di Ferrara, I-44122 Ferrara, Italy \\ ${ }^{15}$ Laboratoire de physique théorique, Département de physique de l'ENS, École normale supérieure, PSL Research University, \\ Sorbonne Université, CNRS, 75005 Paris, France \\ ${ }^{16}$ Department of Physics and Soft and Living Matter Program, Syracuse University, Syracuse, New York 13244, USA
}

(Received 12 March 2018; published 28 June 2018)

\begin{abstract}
Experiments on spin glasses can now make precise measurements of the exponent $z(T)$ governing the growth of glassy domains, while our computational capabilities allow us to make quantitative predictions for experimental scales. However, experimental and numerical values for $z(T)$ have differed. We use new simulations on the Janus II computer to resolve this discrepancy, finding a time-dependent $z\left(T, t_{w}\right)$, which leads to the experimental value through mild extrapolations. Furthermore, theoretical insight is gained by studying a crossover between the $T=T_{c}$ and $T=0$ fixed points.
\end{abstract}

DOI: 10.1103/PhysRevLett.120.267203

The study of spin glasses (SGs) [1,2] has long been a key problem in statistical mechanics, providing ideas that have born fruit in fields as diverse as econophysics, biology, or optimization in computer science. From a fundamental point of view, SGs are paradigmatic as the most approachable model for glassy behavior, both experimentally and theoretically. However, despite this relative simplicity, SG experiments and theory have traditionally developed separately, for practical and conceptual reasons. On the one hand, numerical simulations were not long enough to reach experimental times, while experiments were not precise enough or even able to measure key physical quantities. On the other hand, experimental samples are perennially out of equilibrium, while theory mostly focuses on the (unreachable) equilibrium phase.
In a typical experiment, the system is rapidly cooled to a subcritical working temperature $T<T_{c}$ and its off-equilibrium evolution (aging) studied. As the waiting time $t_{w}$ increases, the size of the glassy domains is seen to grow as $\xi\left(t_{w}\right) \propto t_{w}^{1 / z(T)}$, with an exponent that is expected to behave as $z(T) \simeq z\left(T_{c}\right) T_{c} / T$ [3]. In traditional experiments [4], based on the shift of the peak in the relaxation rate $S\left(t_{w}\right)$, $z(T)$ was difficult to measure. Fortunately, the availability of excellent samples with a film geometry has suggested a new approach to the precision measurement of $z_{c}=$ $z(T) T / T_{c}$ [5]. The time that $\xi\left(t_{w}\right)$ needs to saturate to the film thickness relates to the activation energies $\Delta_{\max }$ $[6,7]$. Varying the film thickness from 9 to $20 \mathrm{~nm}$ resulted in the measurement $z_{c} \approx 9.62$ [5], very far from the value predicted by numerical simulations $z_{c}=6.86(16)$ [8], $z_{c}=6.80(15)$ [9]. 
Fortunately, recent theoretical progress makes it feasible to address the above-mentioned disagreement. A key development has been the introduction of the Janus $[10,11]$ and Janus II [12] computers, which have extended the numerical exploration of the dynamics almost to the experimental scale $[8,13]$. In addition, the introduction of quantitative staticsdynamics dictionaries (first based on microscopic quantities $[8,14,15]$ and more recently on experimentally measurable features [13]) has clarified the relevance of the equilibrium phase for the off-equilibrium dynamics and showed how to extrapolate simulations to the experimental scale. Finally, the (macroscopic) experimental measurement of the size of glassy domains was shown to be consistent with the (microscopic) definition based on correlation functions [16].

Here, we resolve the discrepancy in $z_{c}$ by finding a (very mild) scale dependence in the dynamical exponent $z\left(T, \xi\left(t_{w}\right)\right)$. We first recognize that time should be traded by length scales. Gentle extrapolations to the relevant experimental scales of $20 \mathrm{~nm}$ [5] then reconcile the numerical and experimental measurements. Such a computation has been possible only because of new data with unprecedented precision, achieved by reducing the uncertainty due to thermal fluctuations, an issue that was typically neglected in previous numerical work. From the theoretical point of view, our study is based on a characterization of the crossover between critical and lowtemperature behavior. This is a very important point, since it resolves a theoretical controversy on how low a temperature must be studied to be free of critical effects, with some authors choosing to work at very low $T$ at the expense of the system sizes that it is possible to equilibrate (e.g., [17]) and others trying to find a tradeoff between temperature and system size (e.g., [14]).

We consider the standard Edwards-Anderson model [18], defined on a three-dimensional cubic lattice of side $L=160$, on whose nodes we place spins $S_{x}= \pm 1$ that interact with their lattice nearest neighbors through

$$
\mathcal{H}=-\sum_{\langle x, y\rangle} J_{x y} S_{x} S_{y}
$$

For each disorder realization $\left\{J_{x y}\right\}$ (a sample), each of the quenched couplings $J_{x y}$ is \pm 1 with $50 \%$ probability. We shall refer to thin CuMn films [5], where the film thickness of $20 \mathrm{~nm}$ translates to a distance of 38 lattice spacings (the typical Mn-Mn distance is $5.3 \AA$ ).

Our systems are initialized with random orientations for the spins (representing a very high starting temperature) and immediately quenched to the working temperature $T<T_{c}=1.102(3)$ [19]. We then follow the evolution with the waiting time $t_{w}$ (measured in units of full lattice sweeps) at constant temperature. For each sample $\left\{J_{x y}\right\}$, we simulate $N_{R}$ real replicas, evolving with different thermal noise. We estimate our statistical errors with a jackknife method [20] (including fit parameters [21]).
Our basic observable is the spatial autocorrelation of the overlap field (discussed in detail in [22]),

$$
\begin{gathered}
C_{4}\left(T, \boldsymbol{r}, t_{w}\right)=\overline{\left\langle q^{(a, b)}\left(\boldsymbol{x}, t_{w}\right) q^{(a, b)}\left(\boldsymbol{x}+\boldsymbol{r}, t_{w}\right)\right\rangle_{T}}, \\
q^{(a, b)}\left(\boldsymbol{x}, t_{w}\right)=S^{(a)}\left(\boldsymbol{x}, t_{w}\right) S^{(b)}\left(\boldsymbol{x}, t_{w}\right) .
\end{gathered}
$$

In these equations, the indices $(a, b)$ label the different real replicas; $\langle\cdots\rangle_{T}$ is the average over the thermal noise [in practice, an average over the $(a, b)$ pairs] and $\overline{(\cdots)}$ is the average over the disorder. In equilibrium simulations, by far the main source of error are the sample-to-sample fluctuations. Therefore, it has been customary to simulate the smallest $N_{R}$ that permits definitions such as (2) and maximize the number $N_{S}$ of samples. Instead, we have $N_{R}=256$ and $N_{S}=16$. This choice, motivated to facilitate future studies of temperature chaos [23], has proven crucial: contrary to our expectations, the increase in $N_{R}$ has produced a dramatic reduction of statistical errors (see the Supplemental Material, SM, [24]). As a result, we have been able to follow the decay of $C_{4}\left(T, r, t_{w}\right)$ over six decades (see inset to Fig. 1). A similar dramatic error reduction with high $N_{R}$ has also been seen in studies of the Gardner transition in structural glasses [25,26].

These correlation functions decay with distance as

$$
C_{4}\left(T, r, t_{w}\right)=r^{-\theta} f\left(r / \xi\left(T, t_{w}\right)\right),
$$

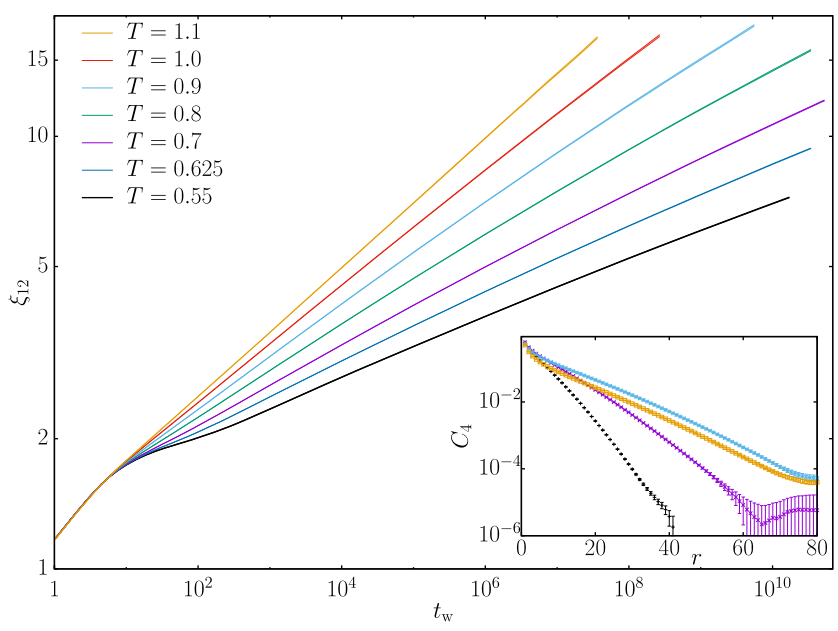

FIG. 1. Growth of the coherence length $\xi_{12}\left(T, t_{w}\right)$ with the waiting time $t_{w}$ after a quench to temperature $T$ in a log-log scale [the critical temperature is $T_{c}=1.102(3)$ ]. Given the smallness of the statistical errors, instead of error bars we have plotted two lines for each $T$, which enclose the error estimate. At this scale, the curves seem linear for long times, indicating a power-law growth but, see Fig. 2, there is actually a measurable curvature. Inset: Spatial autocorrelation function of the overlap field $C_{4}\left(T, r, t_{w}\right)$, plotted as a function of distance at the last simulated time for several temperatures. Note the six orders of magnitude in the vertical axis. 
so the growing $\xi$ can be computed through integral estimators [8,22]: $I_{k}\left(T, t_{w}\right)=\int_{0}^{\infty} d r r^{k} C_{4}\left(T, r, t_{w}\right)$. Then, $\xi_{k, k+1}\left(T, t_{w}\right)=I_{k+1}\left(T, t_{w}\right) / I_{k}\left(T, t_{w}\right)$. As in recent work [13,16,22,27], we use $k=1$ (see [28] for technical details). The resulting $\xi_{12}$ is plotted in Fig. 1 for all our working temperatures. The numerical $[8,22,27]$ and experimental [5] state of the art describes the growth of $\xi_{12}$ with a power law,

$$
\xi_{12}\left(T, t_{w}\right) \simeq A(T) t_{w}^{1 / z(T)} .
$$

However, with our increased precision, (5) is no longer a faithful representation of the dynamics. Indeed, if we switch to $x=\log \xi_{12}$ as the independent variable, we can interpolate our data as

$$
\log t_{w}\left(T, \xi_{12}\right)=c_{0}(T)+c_{1}(T) x+c_{2}(T) x^{2} .
$$

Notice that $c_{2}=0$ would reduce to (5), while $c_{2}>0$ would indicate a slowing down of the dynamics for increasing $\xi_{12}$. Indeed, see Fig. 2, we find that $c_{2}$ vanishes only at $T=T_{c}$, with $z_{c}=z\left(T=T_{c}\right)=6.69(6)$ [29]. Of course, (6), useful as an interpolation, is not suitable to extrapolate for longer times than simulated. In order to do that, we need some insight from theory [30].

We can gain much insight into the SG phase by considering the algebraic prefactor in (4), determined by an exponent $\theta$. At $T_{c}, \theta=1+\eta$, where $\eta=-0.390(4)$ [19] is the anomalous dimension. In the SG phase, there are differing expectations for $\theta$ in the two main theoretical pictures. The droplet description [31-33] expects coarsening domains, and therefore, $\theta=0$. On the other hand, the replica symmetry breaking (RSB) theory expects spacefilling domains where $C_{4}$ vanishes at constant $r / \xi_{12}$ as $t_{w}$ grows. In particular, $\theta$ is given by the replicon, a critical mode analogous to magnons in Heisenberg ferromagnets (see [15] for a detailed discussion). The best previous numerical study of $\theta$ [22], found $\theta=0.38(2)$, with a small

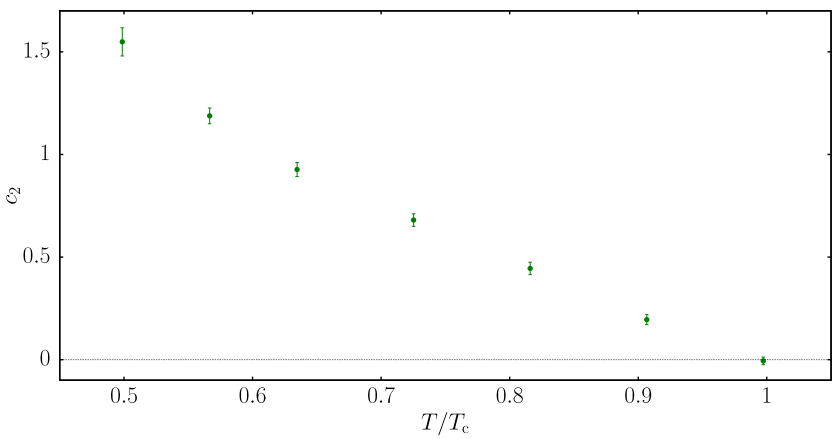

FIG. 2. Deviation of $\xi_{12}\left(t_{w}\right)$ from a simple power-law growth. We plot the quadratic parameter $c_{2}$ in a fit to (6) (see the SM [24] for fitting parameters). This quantity is zero at the critical point but has a positive value at low temperatures, indicating that the growth of $\xi_{12}$ slows down over the simulated time range.
$T$ dependence that was vaguely attributed to the effect of the critical point.

We can obtain $\theta$ by noticing that $I_{2}\left(T, \xi_{12}\right) \propto \xi_{12}^{3-\theta}$. However, again we find that while $\theta\left(T_{c}\right)$ is compatible with $1+\eta$, for $T<T_{c}$ we actually have $\theta\left(T, \xi_{12}\right)$, slowly decreasing as $\xi_{12}$ increases (or $T$ decreases). This may seem an unsatisfactory result, since, in the large- $\xi_{12}$ limit, $\theta\left(T, \xi_{12}\right)$ should tend to a $T$-independent constant (possibly zero). The simplest explanation is that low values of $\xi_{12}$ are affected by the $T=T_{c}$ fixed point with $\theta \approx 0.61$ [an idea supported by the higher measured $\theta\left(T, \xi_{12}\right)$ for the higher $\left.T\right]$, while for $\xi_{12} \rightarrow \infty$ we should see a crossover to the $T=0$ fixed point, with an unknown $\theta(T=0)$ (see also [27]).

In analogy with the ferromagnetic phase of the $O(N)$ model, we can model this crossover in terms of a Josephson length $\ell_{J}$ [34]. Close to $T_{c}$, this should grow as $\ell_{J} \propto\left(T_{c}-T\right)^{-\nu}$, with $\nu=2.56(4)$ [19], while scaling corrections are expected for the lowest temperatures [35]. If this hypothesis is correct, our data for different temperatures should come together when plotted in terms of a scaling variable $x=\ell_{J} / \xi_{12}$. We test this scaling in the inset to Fig. 3, where we consider the ratio $\xi_{23} / \xi_{12}$ between two different determinations of the coherence length, which should be scale invariant in the large- $\xi_{12}$ limit (different definitions of $\xi$ all grow at the same rate but differ in a small constant factor, see Fig. 4 in [22]). As expected, there is an enveloping curve for the data at different $T$. In particular, the curves for $T=0.55,0.625,0.7$ appear free from the influence of the critical point.

Similarly, $\theta\left(T, \xi_{12}\right)=3-d \log I_{2} / d \log \xi_{12}$, which we can compute numerically (see the SM [24]), turns out to be a function of $x$, see the collapsing curve in Fig. 3. We are interested in estimating $\theta(x)$ at the experimentally relevant scale of $\xi_{\text {films }}=38$ for thin films, recall our discussion of (1). As discussed, the RSB and droplet pictures have diverging expectations for $\theta(0)$, that is, for the $\xi_{12} \rightarrow \infty$ limit, so we can use them as upper and lower bounds. In RSB theory, see SM [24] and Fig. 3, we can compute an extrapolation to $\theta(0) \approx 0.30$, although we take $\theta^{\text {upper }}=$ 0.35 as our upper bound for $\theta$. In the droplet description, we expect $\theta(x)=C x^{\zeta}$, where $\zeta$ is, in principle, the stiffness exponent $\zeta \approx 0.24$ [36]. As in [14], we find that the droplet behavior can be reached in the infinite- $\xi_{12}$ limit but only with a smaller exponent $\zeta \approx 0.15$, which, furthermore, is highly sensitive to the fitting range. Using the droplet extrapolation for $\xi_{\text {films }}=38$, we obtain $\theta\left(\xi_{\text {films }}\right) \approx 0.28$. Since our microscopically determined $\xi_{12}$ may differ by a small constant factor from a macroscopic measurement of $\xi$ [16], we have also considered $\xi_{\text {films }}=76$, which brings the exponent down to $\theta\left(\xi_{\text {films }}\right)=0.25$ (see Fig. 3). In short, as observed in previous work $[14,15]$, for the experimentally relevant scale, the physics is well described by a noncoarsening picture, with $0.25<\theta\left(\xi_{\text {films }}\right)<0.35$ depending on the theory we use to extrapolate the data and the exact value chosen for the experimental scale. 


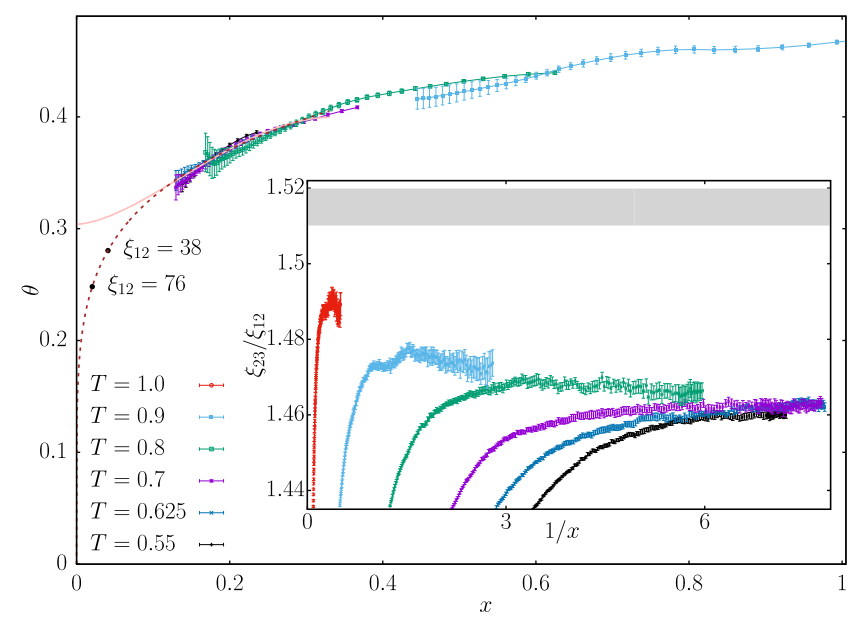

FIG. 3. Crossover between the $T=T_{c}$ and the $T=0$ fixed points controlled by a Josephson length $\ell_{J}(T)$, with $\ell_{J}(T) \propto$ $\left(T_{c}-T\right)^{-\nu}$ close to $T_{c}$ see text). The relevant scaling variable is $x=\ell_{J}(T) / \xi_{12}$. The inset considers the ratio $\xi_{23} / \xi_{12}$ between two definitions of the coherence length, which should be constant in the large- $\xi_{12}$ (or $x \rightarrow 0$ ) limit. For $T$ close to $T_{c}$, this ratio initially grows, approaching the $T=T_{c}$ value (represented by the thick gray line) and eventually relaxes towards the $T=0$ fixed point. Main plot: Evolution of the replicon exponent $\theta$ for several temperatures. We show two possible extrapolations to infinite $\xi_{12}$ : one with finite $\theta$, as expected in the RSB picture, and one with $\theta=0$, as expected in the droplet picture. For the latter, we also show the extrapolated value for the experimental scale corresponding to experiments in CuMn films [5], which we estimate between $\xi_{12}=38$ and $\xi_{12}=76$.

As discussed in the Introduction, experiments observe a constant $z(T) T / T_{c} \approx 9.62$ [5]. In the previous discussion, on the other hand, we have found $z_{c}=6.69(6)$ and a growing $z\left(T, \xi_{12}\right)$. Therefore, in order to compare our results with experiments, the first step is finding some way to extrapolate for $\xi_{12}=\xi_{\text {films }}$. The most natural possibility, given the smoothness of the data in Fig. 1, is to assume that $t_{w}=A(T) \xi_{12}^{z\left(T, \xi_{12}\right)}$, with a $z\left(T, \xi_{12}\right)$ that tends to a finite $z_{\infty}(T)$ when $\xi_{12} \rightarrow \infty, z\left(T, \xi_{12}\right)-z_{\infty}(T) \propto \xi_{12}^{-\omega}$, thus

$$
\log t_{w}=D(T)+z_{\infty}(T) \log \xi_{12}+E(T) \xi_{12}^{-\omega}
$$

where $\omega$ is the exponent that controls finite- $\xi_{12}\left(t_{\mathrm{w}}\right)$ corrections. At $T_{c}$, we expect $\omega=1.12(10)[9,19,27]$. For $T<T_{c}$, the leading behavior is given by $\omega=\theta$ ([15] and Sec. 4.2 in [14]). When fitting to (7), in principle, one must consider possible systematic effects from the fitting range $\xi_{12} \geq \xi_{12}^{\min }$ and the increased statistical error due to our uncertainty in the value of $\theta$. However, see SM [24], these effects have little impact on our final estimates.

An alternative interpretation is to consider a crossover to activated dynamics, as proposed by the Saclay group
[37,38]. Free-energy barriers are considered from a dynamical point of view, with a growth exponent $\Psi$,

$$
\log t_{w}=F(T)+z_{c} \log \xi_{12}+G(T) \xi_{12}^{\Psi},
$$

hence $\quad z\left(T, \xi_{12}\right)=d \log t_{w} / d \log \xi_{12}=z_{c}+G(T) \Psi \xi_{12}^{\Psi}$. Equation (8) is a refinement of droplet theory [33] and has been used before in experiments [39] and simulations [40] with values of $\Psi \approx 1$ [41]. RSB theory is neutral with respect to choosing Ansätze (7) or (8). We recall the numerical result in infinite dimensions [42] of $\tau \sim$ $\exp \left(-N^{b}\right)$ for the timescales associated with the largest energy barriers, with $b \approx 1 / 3$ (see also [43,44]). This result can be connected with finite $D$ at the upper critical dimensions $D_{u}=6$, which yields $\Psi\left(D_{u}\right)=6 b$. We note, in particular, that (7) can be regarded as a $\Psi \rightarrow 0$ limit of (8). With previous data, it was not possible to distinguish the behavior of (8) and that of a simple power law [22]. With the present simulations, we find that (8) also yields good fits for $t_{w}\left(\xi_{12}\right)$, with $\Psi \approx 0.4$ (again, the dependence on the fitting range is minimal, see SM [24]).

Therefore, both (7) and (8) can explain the behavior of the data for the simulated scales. In order to see whether they are useful to explain the experiments, we consider the quantity $Z_{c}(T)=z\left(T, \xi_{\text {films }}\right) T / T_{c}$, where $z\left(T, \xi_{\text {films }}\right)$ is the derivative of either (7) or (8) at $\xi_{\text {films. }}$. The result is plotted in Fig. 4 (see SM [24] for the full fit parameters). Remarkably, the convergent Ansatz of (7) produces an almost constant

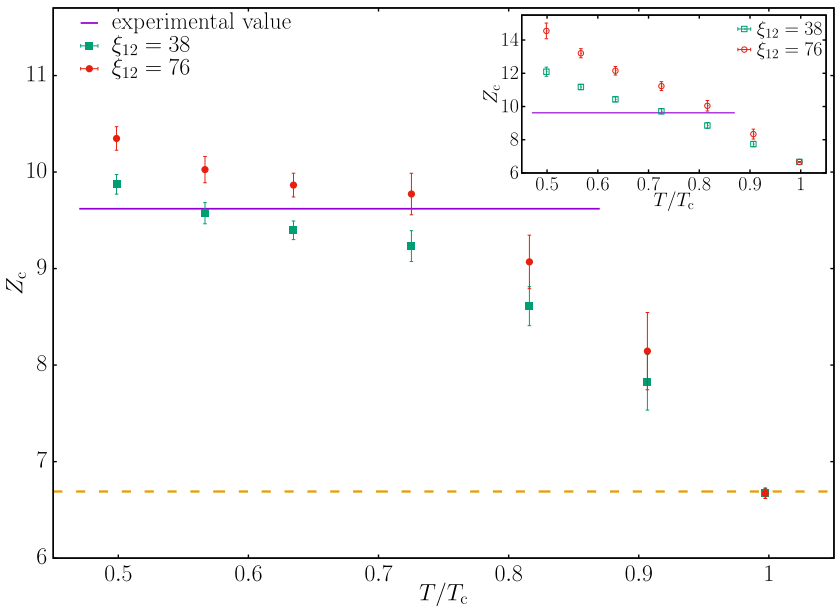

FIG. 4. Value of the experimental aging rate for SGs $Z_{c}(T)=z\left(T, \xi_{\text {films }}\right) T / T_{c}$, extrapolated from our data for values of the coherence length corresponding to thin CuMn films. The main plot considers an Ansatz (7) with a finite $z\left(T, \xi_{12} \rightarrow \infty\right)$, which agrees very well with the experimental value of $Z_{c}(T) \approx$ 9.62 [5], indicated by the straight line, whose width represents the experimental temperature range. Notice that critical effects are only visible for $T>0.7$. Inset: Same plot but now considering a crossover to activated dynamics (8), as in [37]. This is less successful at reproducing the roughly constant $Z_{c}(T)$ observed in experiments. 
$Z_{c}$ in a wide $T$ range, which additionally fits well the experimental value of $Z_{c} \approx 9.62$. The activated dynamics of (8), on the other hand, are not a good fit for the experimental behavior (inset to Fig. 4).

Using simulations for very large systems with many replicas on Janus II, we have found that the growth of the SG coherence length is controlled by a time-dependent $z\left(T, \xi\left(t_{w}\right)\right)$ exponent. After describing the dynamics as governed by a crossover between a critical and a lowtemperature fixed point, we have been able to model this growth quantitatively and to extrapolate to experimental length scales. The resulting exponent is consistent with the most recent experimental measurements for power-law dynamics. In addition, we find clear evidence of noncoarsening dynamics at the experimental scale and find that temperatures $T \lesssim 0.7$ are free of critical effects and therefore safe for numerical studies of the SG phase.

An open question concerns the generality of these results. Indeed, CuMn is a Heisenberg, rather than Ising, SG. However, even the purest Heisenberg system has unavoidable anisotropies, such as Dzyaloshinsky-Moriya interactions [45]. These interactions, though tiny, extend over dozens of lattice spacings, which magnifies their effect. In fact, we know that Ising is the ruling universality class in the presence of coupling anisotropies [46]. We also remark that high-quality measurements on GeMn are excellently fit with Ising scaling laws [7]. Our results also match the most recent and accurate measurements on CuMn [5].

More generally, this study is a clear demonstration of the importance of high-precision results for the investigation of glassiness. Indeed, reducing the errors has shown that the aging rate slows down during the dynamics, contrary to previous findings. A similar change of paradigm might happen for structural glasses.

We thank R. Orbach for encouraging discussions. This work was partially supported by Ministerio de Economía, Industria y Competitividad (MINECO) (Spain) through Grants No. FIS2013-42840-P, No. FIS2015-65078-C2, No. FIS2016-76359-P, and No. TEC2016-78358-R, by the Junta de Extremadura (Spain) through Grant No. GRU10158 (partially funded by FEDER) and by the DGA-FSE (Diputación General de Aragón-Fondo Social Europeo). This project has received funding from the European Research Council (ERC) under the European Union's Horizon 2020 research and innovation program (Grants No. 694925 and No. 723955-GlassUniversality). D. Y. acknowledges support by the Soft and Living Matter Program at Syracuse University.

*dyllanes@syr.edu

[1] J. A. Mydosh, Spin Glasses: An Experimental Introduction (Taylor and Francis, London, 1993).

[2] A. P. Young, Spin Glasses and Random Fields (World Scientific, Singapore, 1998).
[3] E. Marinari, G. Parisi, F. Ricci-Tersenghi, J. J. RuizLorenzo, and F. Zuliani, J. Stat. Phys. 98, 973 (2000).

[4] Y. G. Joh, R. Orbach, G. G. Wood, J. Hammann, and E. Vincent, Phys. Rev. Lett. 82, 438 (1999).

[5] Q. Zhai, D. C. Harrison, D. Tennant, E. D. Dalhberg, G. G. Kenning, and R. L. Orbach, Phys. Rev. B 95, 054304 (2017).

[6] S. Guchhait and R. Orbach, Phys. Rev. Lett. 112, 126401 (2014).

[7] S. Guchhait and R. L. Orbach, Phys. Rev. Lett. 118, 157203 (2017)

[8] F. Belletti, M. Cotallo, A. Cruz, L. A. Fernandez, A. GordilloGuerrero, M. Guidetti, A. Maiorano, F. Mantovani, E. Marinari, V. Martín-Mayor, A. M. Sudupe, D. Navarro, G. Parisi, S. Perez-Gaviro, J. J. Ruiz-Lorenzo, S. F. Schifano, D. Sciretti, A. Tarancon, R. Tripiccione, and J. L. Velasco et al. (Janus Collaboration), Phys. Rev. Lett. 101, 157201 (2008).

[9] M. Lulli, G. Parisi, and A. Pelissetto, Phys. Rev. E 93, 032126 (2016).

[10] F. Belletti, M. Guidetti, A. Maiorano, F. Mantovani, S. F. Schifano, R. Tripiccione, M. Cotallo, S. Perez-Gaviro, D. Sciretti, J. L. Velasco, A. Cruz, D. Navarro, A. Tarancon, L. A. Fernandez, V. Martín-Mayor, A. Muñoz-Sudupe, D. Yllanes, A. Gordillo-Guerrero, J. J. Ruiz-Lorenzo, and E. Marinari et al. (Janus Collaboration), Comput. Sci. Eng. 11, 48 (2009).

[11] M. Baity-Jesi, R. A. Baños, A. Cruz, L. A. Fernandez, J. M. Gil-Narvion, A. Gordillo-Guerrero, M. Guidetti, D. Iniguez, A. Maiorano, F. Mantovani, E. Marinari, V. Martín-Mayor, J. Monforte-Garcia, A. Munoz Sudupe, D. Navarro, G. Parisi, M. Pivanti, S. Perez-Gaviro, F. Ricci-Tersenghi, and J. J. Ruiz-Lorenzo et al., Eur. Phys. J. Spec. Top. 210, 33 (2012).

[12] M. Baity-Jesi, R. A. Baños, A. Cruz, L. A. Fernandez, J. M. Gil-Narvion, A. Gordillo-Guerrero, D. Iniguez, A. Maiorano, F. Mantovani, E. Marinari, V. Martín-Mayor, J. Monforte-Garcia, A. Muñoz Sudupe, D. Navarro, G. Parisi, S. Perez-Gaviro, M. Pivanti, F. Ricci-Tersenghi, J. J. RuizLorenzo, and S. F. Schifano et al. (Janus Collaboration), Comput. Phys. Commun. 185, 550 (2014).

[13] M. Baity-Jesi, E. Calore, A. Cruz, L. A. Fernandez, J. M. Gil-Narvión, A. Gordillo-Guerrero, D. Iñiguez, A. Maiorano, E. Marinari, V. Martin-Mayor, J. Monforte-Garcia, A. Muñoz Sudupe, D. Navarro, G. Parisi, S. Perez-Gaviro, F. RicciTersenghi, J. J. Ruiz-Lorenzo, S. F. Schifano, B. Seoane, and A. Tarancón et al., Proc. Natl. Acad. Sci. U.S.A. 114, 1838 (2017).

[14] R. A. Baños, A. Cruz, L. A. Fernandez, J. M. Gil-Narvion, A. Gordillo-Guerrero, M. Guidetti, A. Maiorano, F. Mantovani, E. Marinari, V. Martín-Mayor, J. MonforteGarcia, A. Muñoz Sudupe, D. Navarro, G. Parisi, S. PerezGaviro, J. J. Ruiz-Lorenzo, S. F. Schifano, B. Seoane, A. Tarancon, and R. Tripiccione et al. (Janus Collaboration), J. Stat. Mech. (2010) P06026.

[15] R. A. Baños, A. Cruz, L. A. Fernandez, J. M. Gil-Narvion, A. Gordillo-Guerrero, M. Guidetti, A. Maiorano, F. Mantovani, E. Marinari, V. Martín-Mayor, J. MonforteGarcia, A. Muñoz Sudupe, D. Navarro, G. Parisi, S. PerezGaviro, J. J. Ruiz-Lorenzo, S. F. Schifano, B. Seoane, A. 
Tarancon, and R. Tripiccione et al. (Janus Collaboration), Phys. Rev. Lett. 105, 177202 (2010).

[16] M. Baity-Jesi, E. Calore, A. Cruz, L. A. Fernandez, J. M. Gil-Narvion, A. Gordillo-Guerrero, D. Iñiguez, A. Maiorano, E. Marinari, V. Martin-Mayor, J. MonforteGarcia, A. Muñoz Sudupe, D. Navarro, G. Parisi, S. Perez-Gaviro, F. Ricci-Tersenghi, J. J. Ruiz-Lorenzo, S. F. Schifano, B. Seoane, and A. Tarancon et al. (Janus Collaboration), Phys. Rev. Lett. 118, 157202 (2017).

[17] W. Wang, J. Machta, H. Munoz-Bauza, and H. G. Katzgraber, Phys. Rev. B 96, 184417 (2017).

[18] S. F. Edwards and P. W. Anderson, J. Phys. F 5, 965 (1975).

[19] M. Baity-Jesi, R. A. Baños, A. Cruz, L. A. Fernandez, J. M. Gil-Narvion, A. Gordillo-Guerrero, D. Iniguez, A. Maiorano, F. Mantovani, E. Marinari, V. Martín-Mayor, J. Monforte-Garcia, A. Muñoz Sudupe, D. Navarro, G. Parisi, S. Perez-Gaviro, M. Pivanti, F. Ricci-Tersenghi, J. J. RuizLorenzo, and S. F. Schifano et al. (Janus Collaboration), Phys. Rev. B 88, 224416 (2013).

[20] D. J. Amit and V. Martín-Mayor, Field Theory, The Renormalization Group and Critical Phenomena, 3rd ed. (World Scientific, Singapore, 2005).

[21] D. Yllanes, Rugged Free-Energy Landscapes in Disordered Spin Systems, Ph.D. thesis, Universidad Complutense de Madrid (2011).

[22] F. Belletti, A. Cruz, L. A. Fernandez, A. Gordillo-Guerrero, M. Guidetti, A. Maiorano, F. Mantovani, E. Marinari, V. Martín-Mayor, J. Monforte, A. Muñoz Sudupe, D. Navarro, G. Parisi, S. Perez-Gaviro, J. J. Ruiz-Lorenzo, S. F. Schifano, D. Sciretti, A. Tarancon, R. Tripiccione, and D. Yllanes (Janus Collaboration), J. Stat. Phys. 135, 1121 (2009).

[23] Janus Collaboration (to be published).

[24] See Supplemental Material at http://link.aps.org/ supplemental/10.1103/PhysRevLett.120.267203 for technical details of our simulations and statistical analysis.

[25] L. Berthier, P. Charbonneau, Y. Jin, G. Parisi, B. Seoane, and F. Zamponi, Proc. Natl. Acad. Sci. U.S.A. 113, 8397 (2016).

[26] B. Seoane and F. Zamponi, Soft Matter DOI: 10.1039/ C8SM00859K (2018).

[27] L. A. Fernández and V. Martín-Mayor, Phys. Rev. B 91, 174202 (2015).
[28] L. A. Fernández, E. Marinari, V. Martin-Mayor, G. Parisi, and J. J. Ruiz-Lorenzo, arXiv:1805.06738.

[29] Our result $z_{c}=z\left(T=T_{c}\right)=6.69(6)$ is significantly more accurate than $z_{c}=6.80(15)$ [9] and $z_{c}=6.86(16)$ [8], even though, unlike Ref. [8], we allow for corrections to scaling, which increases the statistical error, see SM [24].

[30] A naive explanation for the curvature in $\xi_{12}\left(T, t_{w}\right)$ would be the existence of finite-size effects (see [8]). However, $c_{2}$ grows as we decrease $T$, while finite-size effects would be controlled by $\xi_{12} / L$, which is smaller for the lower temperatures. See SM [24] for extensive checks that our $L=160$ are safe.

[31] W. L. McMillan, Phys. Rev. B 28, 5216 (1983).

[32] A. J. Bray and M. A. Moore, Phys. Rev. Lett. 41, 1068 (1978).

[33] D. S. Fisher and D. A. Huse, Phys. Rev. Lett. 56, 1601 (1986).

[34] B. D. Josephson, Phys. Lett. 21, 608 (1966).

[35] In theory, $\ell_{J} \propto\left[1+j_{0}\left(T_{c}-T\right)^{\nu}+j_{1}\left(T_{c}-T\right)^{\omega \nu}\right]\left(T_{c}-T\right)^{-\nu}$, where we include analytic $\left(j_{0}\right)$ and confluent $\left(j_{1}\right)$ scaling corrections with $\omega=1.12(10)$ [19]. For Fig. 3, we have chosen $j_{0}$ and $j_{1}$ to obtain the best collapse for the lowest temperatures.

[36] S. Boettcher, Eur. Phys. J. B 38, 83 (2004).

[37] J.-P. Bouchaud, V. Dupuis, J. Hammann, and E. Vincent, Phys. Rev. B 65, 024439 (2001).

[38] L. Berthier and J.-P. Bouchaud, Phys. Rev. B 66, 054404 (2002).

[39] A. G. Schins, A. F. M. Arts, and H. W. de Wijn, Phys. Rev. Lett. 70, 2340 (1993).

[40] H. Rieger, J. Phys. A 26, L615 (1993).

[41] $G(T)$ in (8) goes to zero at $T_{c}$ as $\left(T_{c}-T\right)^{\Psi_{\nu}}$, which is another form of the Josephson scaling.

[42] A. Billoire and E. Marinari, J. Phys. A 34, L727 (2001).

[43] G. Rogers and M. A. Moore, J. Phys. A 22, 1085 (1989).

[44] S. G. W. Colborne, J. Phys. A 23, 4013 (1990).

[45] A. J. Bray and M. A. Moore, J. Phys. C 15, 3897 (1982).

[46] M. Baity-Jesi, L. A. Fernandez, V. Martín-Mayor, and J. M. Sanz, Phys. Rev. 89, 014202 (2014). 